\title{
Coulomb Energy of Nuclei
}

\author{
V.R. Shaginyan * \\ Petersburg Nuclear Physics Institute, Gatchina 188350, Russia
}

\begin{abstract}
The density functional determining the Coulomb energy of nuclei is calculated to the first order in $e^{2}$. It is shown that the Coulomb energy includes three terms: the Hartree energy; the Fock energy; and the correlation Coulomb energy (CCE), which contributes considerably to the surface energy, the mass difference between mirror nuclei, and the single-particle spectrum. A CCE-based mechanism of a systematic shift of the single-particle spectrum is proposed. A dominant contribution to the CCE is shown to come from the surface region of nuclei. The CCE effect on the calculated proton drip line is examined, and the maximum charge $Z$ of nuclei near this line is found to decrease by 2 or 3 units. The effect of Coulomb interaction on the effective proton mass is analyzed.
\end{abstract}

PACS numbers: 21.10.Sf, 21.10.Dr, 21.10.-k

*E-mail: vrshag@thd.pnpi.spb.ru 


\section{INTRODUCTION}

Relatively weak Coulomb interaction substantially affects the properties of nuclei owing to its long-range character. It is reliably established that the electrostatic Coulomb energy of the distributed charge is the dominant term in the Coulomb energy of nuclei. This term, also known as the Hartree energy, is proportional to $Z^{2} e^{2} / R$, where $Z$ is the number of intranuclear protons, $R$ is the nuclear radius, and $e$ is the proton charge; at large values of $Z$, it induces the breakup of nuclei. At the same time, there are a few less significant contributions to the Coulomb energy. These include the Fock exchange contribution and a number of terms associated with the correlated motion of nucleons. It will be shown below that, in constructing the density functional determining the Coulomb energy of a nucleus, it is sufficient to calculate all these terms to the first order in $e^{2}$. The presence of these terms is clearly illustrated by the well-known Nolen-Schiffer anomaly [四], which was discovered more than 30 years ago. This anomaly was deduced from a comparison of the calculated mass difference between two mirror nuclei with its experimental value. The comparison revealed that, on average, the results of the calculations performed without taking consistently into account the correlation Coulomb energy (CCE) fall short of relevant experimental values by $10 \%$ [2, 3]. This discrepancy can be removed by introducing charge-dependent forces [4] whose strength is assumed to be preset by this discrepancy. However, this is possible if we are sure that nuclear dynamics is taken properly into account and that all the Coulomb contributions are consistently included. A new mechanism that enhances the contribution of Coulomb interaction to the energy of the nuclear ground state and which is caused by the presence of a surface (a general property of equilibrium finite Fermi systems) was found in [5,6]. This contribution, which is proportional to the nuclear surface $\left(Z^{2 / 3}\right)$, made it possible to explain a dominant part of the above anomaly.

The main objective of this study is to calculate consistently the density functional determining

the Coulomb energy. As the result of this calculation, which will be performed to the first order in $e^{2}$ and which will take into account the mechanism enhancing the CCE contribution, we will be able to clarify the effect of this mechanism on the single-particle spectrum of nuclei, on the effective nucleon mass, and on the position of the proton drip line. In what follows, we do not highlight the difference between this functional and the Coulomb energy unless this leads to confusion.

The ensuing exposition is organized as follows. A general formulation of our approach to calculating the Coulomb energy is given in Section 2. Section 3 is devoted to calculating the systematic CCE-induced shifts of the single-particle spectrum and the proton drip line. The effect of Coulomb interaction on the effective proton mass is considered in Section 4. Section 5 summarizes the basic results.

\section{COULOMB CORRELATION ENERGY}

In order to formulate the aforementioned mechanism and to consider its effect, we will make use of the Hartree-Fock method employing effective forces, which was substantiated within densityfunctional theory [7]. In this case, the energy $E$ of the nuclear ground state has the form

$$
E=F_{0}\left[\rho_{p}, \rho_{n}\right]+F_{c}\left[\rho_{p}, \rho_{n}\right] .
$$

where $F_{0}$ is that part of the functional which is due to nuclear forces. Since we assume that the charge symmetry of nuclear forces takes place, $F_{0}$ depends identically on the single-particle densities $\rho_{p}$ and 
$\rho_{n}$ of protons and neutrons (these densities are determined by minimizing $E$ ). The term $F_{c}$ is due to other interactions, which are weaker than nuclear forces. The smallness of these interactions can be characterized by the ratio a of the potential energy of these interactions between two nucleons to the Fermi energy $\varepsilon_{F} \simeq 40 \mathrm{MeV}$. For the Coulomb interaction, this ratio is $\alpha=e^{2} / r_{0} \varepsilon_{F} \simeq 0.03$, where $r_{0}$ is the mean spacing between nucleons in equilibrium nuclear matter. Therefore, it is sufficient to calculate the functional $F_{c}$ in the first order in the relevant coupling constant. In the case of Coulomb interaction, which is the main subject of the present study, the functional $F_{c}$ will be calculated to the first order in $e_{2}$. This functional could be approximated by some simple expression, as was done, for example, for the functional $F_{0}$. However, experience gained in calculating the mass differences between mirror nuclei (these mass differences are directly determined by the functional $F_{c}$ ) proves the inefficiency of such attempts. Moreover, this oversimplified approximation does not reveal the physics behind the functional $F_{c}$. Therefore, it is preferable to calculate the functional $F_{c}$. In doing this, it is assumed that the functional $F_{0}$ is known. It can be taken in the form specified by the Skyrme interaction [8] or in the form proposed in [9]. The latter is advantageous in that it is characterized by a high precision, is simple in use, and involves the bare nucleon mass. By construction, the functional $F_{0}$ must contain the unnrenormalized mass $M$ of the proton (neutron) rather than its effective mass $M^{*}$ because the effective mass, as well as the nuclear spectrum, cannot be directly included in it [10]. The single-particle distributions of the proton and neutron densities $\left(\rho_{p}\right.$ and $\rho_{n}$, respectively) are specified as

$$
\rho_{p}(\mathbf{r})=\sum_{l} n_{p}^{l}\left|\phi_{p}^{l}(\mathbf{r})\right|^{2} ; \quad \rho_{n}(\mathbf{r})=\sum_{l} n_{n}^{l}\left|\phi_{n}^{l}(\mathbf{r})\right|^{2}
$$

where $n_{p}^{l}$ and $n_{n}^{l}$ are the occupation numbers for single-particle proton and neutron levels, respectively, while $\phi_{p}^{l}$ and $\phi_{n}^{l}$ are, respectively, the proton and neutron single-particle wave functions determined from the Hartree-Fock equation [8]. The term $F_{c}\left[\rho_{p}\right]$ is usually taken in the first order in the Coulomb interaction in the Hartree-Fock approximation [7,8]:

$$
F_{c}=\frac{e^{2}}{2}\left[\int \frac{\rho_{p}\left(\mathbf{r}_{1}\right) \rho_{p}\left(\mathbf{r}_{2}\right)}{\left|\mathbf{r}_{1}-\mathbf{r}_{2}\right|} d \mathbf{r}_{1} d \mathbf{r}_{2}-\int\left[\frac{\chi_{p}^{0}\left(\mathbf{r}_{1}, \mathbf{r}_{2}, i \omega\right)+2 \pi \rho_{p}\left(\mathbf{r}_{1}\right) \delta\left(\mathbf{r}_{1}-\mathbf{r}_{2}\right) \delta(\omega)}{\left|\mathbf{r}_{1}-\mathbf{r}_{2}\right|}\right] \frac{d \mathbf{r}_{1} d \mathbf{r}_{2} d \omega}{2 \pi}\right] .
$$

Here, $\chi_{p}^{0}\left(\mathbf{r}_{1}, \mathbf{r}_{2}, \omega\right)$ is the linear response function for noninteracting protons moving in the selfconsistent single-particle field $V_{p}$. The first and the second term on the right-hand side of Eq. (3) are, respectively, the Hartree and the Fock density functional $\left(F_{c}^{H}\right.$ and $F_{c}^{F}$ ). The exchange term is usually approximated by the expression [8],

$$
F_{c}^{F}=-\frac{3 e^{2}}{4}\left(\frac{3}{\pi}\right)^{1 / 3} \int \rho_{p}^{4 / 3}(\mathbf{r}) d \mathbf{r} .
$$

However, the equality in (3) is not exact even in the first order in the Coulomb interaction $\left(e^{2}\right.$ order $)$ because it does not include the functional $F_{c}^{c o r r}\left[\rho_{p}(\mathbf{r})\right]$ taking into account the contribution to the Coulomb energy from the correlated motion of protons under the effect of the effective (residual) nuclear interaction $R_{\alpha \beta}\left(\mathbf{r}_{1}, \mathbf{r}_{2}\right)$. In the first order in $e^{2}$, the CCE functional $F_{c}^{c o r r}$ has the form [6]

$$
F_{c}^{c o r r}\left[\rho_{p}\right]=-\frac{e^{2}}{2} \int\left[\frac{\chi_{p p}\left(\mathbf{r}_{1}, \mathbf{r}_{2}, i \omega\right)-\chi_{p}^{0}\left(\mathbf{r}_{1}, \mathbf{r}_{2}, i \omega\right)}{\left|\mathbf{r}_{1}-\mathbf{r}_{2}\right|}\right] \frac{d \mathbf{r}_{1} d \mathbf{r}_{2} d \omega}{2 \pi}
$$


where $\chi_{p p}\left(\mathbf{r}_{1}, \mathbf{r}_{2}, \omega\right)$ is the exact function representing the linear response of intranuclear protons to an external electric field. It should be noted that the function $\chi_{p p}$ is completely determined by the functional $F_{0}$ [6] and that it involves no smallness associated with the Coulomb interaction. The total response function contains three independent components $-\chi_{p p}, \chi_{n n}$ and $\chi_{n p}-$ and satisfies the equation

$$
\chi_{l m}=\chi_{l}^{0} \delta_{l m}+\sum_{k} \chi_{l}^{0} R_{l k} \chi_{k m}
$$

where integration signs are omitted for the sake of simplicity. The response function $\chi_{p}^{0}$ for noninteracting nucleons (protons) moving in the self-consistent field $V_{p}$ has the form

$$
\chi_{p}^{0}\left(\mathbf{r}_{1}, \mathbf{r}_{2}, \omega\right)=\sum_{i, k} n_{p}^{i}\left(1-n_{p}^{k}\right) \phi_{p}^{i *}\left(\mathbf{r}_{1}\right) \phi_{p}^{i}\left(\mathbf{r}_{2}\right) \phi_{p}^{k *}\left(\mathbf{r}_{2}\right) \phi_{p}^{k}\left(\mathbf{r}_{1}\right)\left[\frac{1}{\omega-\omega_{p}^{i k}+i \eta}-\frac{1}{\omega+\omega_{p}^{i k}-i \eta}\right] .
$$

The single-particle eigenvalues $\varepsilon_{p}^{i}$, the frequencies $\omega_{p}^{i k}=\varepsilon_{p}^{k}-\varepsilon_{p}^{i}$, the single-particle wave functions $\phi_{p}^{i *}(\mathbf{r})$, and the corresponding densities $\rho_{p}$ and $\rho_{n}$ are determined by solving the single-particle Hartree-Fock-like equations, or the Kohn-Sham equations [10],

$$
\left(-\frac{\nabla^{2}}{2 M}+V_{p}(\mathbf{r})\right) \phi_{p}^{i}(\mathbf{r})=\varepsilon_{p}^{i} \phi_{p}^{i}(\mathbf{r})
$$

which are derived by means of a conventional procedure that amounts to varying Eq. (1) with respect to the single-particle functions $\phi_{n, p}^{l}[0,8]$. The self-consistent potential $V_{p}$ and the effective interaction $R_{\alpha \beta}$ are given by

$$
V_{p}(\mathbf{r})=\frac{\delta\left(F_{0}\left[\rho_{p}, \rho_{n}\right]-T_{0}\left[\rho_{p}, \rho_{n}\right]\right)}{\delta \rho_{p}(\mathbf{r})} ; \quad R_{\alpha \beta}\left(\mathbf{r}_{1}, \mathbf{r}_{2}\right)=\frac{\delta^{2}\left(F_{0}\left[\rho_{p}, \rho_{n}\right]-T_{0}\left[\rho_{p}, \rho_{n}\right]\right)}{\delta \rho_{\alpha}\left(\mathbf{r}_{1}\right) \delta \rho_{\beta}\left(\mathbf{r}_{1}\right)}
$$

where $T_{0}\left[\rho_{p}, \rho_{n}\right]$ is the kinetic-energy functional for noninteracting nucleons. Thus, we conclude that, to the first order in $e^{2}$, the eventual form of the functional $F_{c}$ is

$$
F_{c}=F_{c}^{H}+F_{c}^{F}+F_{c}^{c o r r}
$$

Equations (1), (3), (5), and (10) provide a basis for consistently calculating the Coulomb energy and for taking into account the Coulomb interaction effect on the properties of nuclei. The poles of the response function c $\chi_{p p}$ determine the collective spectrum of nuclei, while the residues at these poles govern the relevant transition probabilities. As can be seen from Eq. (5), a dominant contribution to $F_{c}^{c o r r}$ comes from collective isoscalar surface vibrations whose excitation energies are much lower than those of corresponding isovector modes. Thus, the functional $F_{c}^{c o r r}$ is controlled by the isoscalar components of the effective interaction.

It is instructive to consider the CCE for symmetric infinite nuclear matter [5,6], in which case the relevant equations are considerably simplified. The function c $\chi_{p p}$ assumes the form

$$
\chi_{p p}(q, \omega)=\frac{1}{2}\left[\frac{\chi^{0}(q, \omega)}{1-R_{+}(q, \omega, \rho) \chi^{0}(q, \omega)}+\frac{\chi^{0}(q, \omega)}{1-R_{-}(q, \omega, \rho) \chi^{0}(q, \omega)}\right],
$$


and determines the functional $F_{c}^{c o r r}$ :

$$
F_{c}^{c o r r}\left[\rho_{p}\right]=-2 \pi e^{2} \int\left[\frac{\chi_{p p}(q, i \omega)-\chi_{p}^{0}(q i \omega)}{q^{2}}\right] \frac{d \mathbf{q} d \omega}{(2 \pi)^{4}}
$$

For symmetric matter, we have $\chi_{p}^{0}=\chi_{n}^{0}=\chi^{0} / 2$; the effective interaction $R(\rho)$ is similar to the local interaction $f(\rho)$ between nucleons that was introduced by Migdal [1]:

$$
\frac{p_{F} M}{\pi^{2}} R_{+}(\rho)=f(\rho) ; \frac{p_{F} M}{\pi^{2}} R_{-}(\rho)=f^{\prime}(\rho) .
$$

Considering that the isoscalar amplitude $f(\rho \rightarrow 0)$ is approximately equal to -2.5 and that $p_{F} M / \pi^{2}=\chi^{0}(0, i 0)$, one can see from Eq. (11) that, at relatively low densities corresponding to the surface region of nuclei, the denominator $1-R_{+} \chi^{0}$ vanishes at small values of $q$ and $\omega$. Therefore, the function $\chi_{p p}$ develops poles on the imaginary axis. These poles, which evince instability of low-density nuclear matter [11], lead to the divergence of the integral in Eq. (12), and this prevents a calculation of the CCE at these nuclear-matter densities. We can conclude that the main contribution to this integral comes from the isoscalar response function. From the above, it also follows that the conventional procedure based on calculating various quantities in the local density approximation (LDA) in infinite uniform matter fails in this case. In this connection, it is worth noting that, although an attempt was made in [12] to construct the functional $F_{0}$ within the LDA, this is impossible for the same reason. It is convenient to calculate the CCE in semi-infinite nuclear matter, where there is a region of low-density nuclear matter near the surface. In the generalized LDA, we then obtain [5, 6],

$$
F_{c}^{c o r r}\left[\rho_{p}\right]=\int \rho_{p}(\mathbf{r}) e_{c}(\rho(\mathbf{r})) d \mathbf{r}
$$

where $e_{c}(\rho)$ is the CCE per proton and $\rho_{p}$ is the single-particle proton density. The calculation of the energy $e_{c}\left(\rho_{p}\right)$ for semi-infinite nuclear matter revealed that, at the surface, this energy has a pronounced positive peak, which corresponds to smoothing the divergence in (12) for uniform nuclear matter [6]. It is convenient to approximate the energy $e_{c}$ by the simple expression

$$
e_{c}\left(\rho_{p}(r)\right)=D\left[\frac{\rho_{p}(r)}{\rho_{0}}\left(1-\frac{\rho_{p}(r)}{\rho_{0}}\right)\right]^{4 / 3},
$$

where $2 \rho_{0}=0.16 \mathrm{fm}^{-3}$ is the equilibrium nuclear density and $D \simeq 6 \mathrm{MeV}$. In contrast to the terms $F_{c}^{H}$ and $F_{c}^{F}$, the energy $e_{c}\left(\rho_{p}\right)$, which has a pronounced peak at the nuclear surface, makes a noticeable contribution to the surface tension $\sigma_{c}$,

$$
\sigma_{c}=\int_{-\infty}^{\infty} \rho_{p}(z)\left[e_{c}\left(\rho_{p}(z)\right)-e_{c}\left(\rho_{p}(-\infty)\right)\right] d z
$$

Hence, the Weizsöcker mass formula must be supplemented with the term $\Delta E$ whose contribution to the total binding energy of a nucleus can be represented as

$$
\Delta E=a_{1}^{\prime} Z+a_{2}^{\prime} Z^{2 / 3}
$$


where $a_{1}^{\prime} \simeq-0.1 \mathrm{MeV}$ and $a_{2}^{\prime} \simeq 1.0 \mathrm{MeV}$. Thus, we can see from Eq. (17) that the surface tension has an isovector component. It will be shown below that this is so for the effective mass as well. It follows from Eq. (17) that the surface tension of the proton nuclear Fermi liquid is effectively greater than that of neutron matter. This means that, when protons are added to a nucleus, their binding energy becomes somewhat smaller because of an increase in the surface of the proton liquid component. It will also be proven below that this decrease in the proton binding energy is sufficient for explaining the mass difference between mirror nuclei. Further, the additional surface tension $\sigma_{c}$ and the addition of extra neutrons are expected to induce opposite modifications in the rootmean-square radius $\left\langle r_{p}>\right.$ of the intranuclear-proton distribution: the former would moderate an increase associated with the latter. The preliminary calculations reveal that this could explain the anomalously small increase in the radius $\left\langle r_{p}\right\rangle$ in going over from the ${ }^{40} \mathrm{Ca}$ to the ${ }^{48} \mathrm{Ca}$ nucleus. The contribution $\sigma_{c}$ must also be taken into account in considering fission barriers for heavy nuclei.

\section{SINGLE-PARTICLE SPECTRUM AND THE PROTON DRIP LINE}

Let us calculate the CCE-induced shift of the single-particle proton excitation spectrum $\varepsilon_{p}^{l}$. For this, we will use the well-known Landau equation [13]

$$
\frac{\delta E}{\delta n_{p}^{l}}=\varepsilon_{p}^{l}
$$

It follows from Eqs. (5) and (18) that this shift $\Delta \varepsilon_{p}^{l}$ can be represented as

$$
\Delta \varepsilon_{p}^{l}=\frac{\delta F_{c}^{c o r r}}{\delta n_{p}^{l}}=-\frac{e^{2}}{2} \frac{\delta}{\delta n_{p}^{l}} \int\left[\frac{\chi_{p p}\left(\mathbf{r}_{1}, \mathbf{r}_{2}, i \omega\right)-\chi_{p}^{0}\left(\mathbf{r}_{1}, \mathbf{r}_{2}, i \omega\right)}{\left|\mathbf{r}_{1}-\mathbf{r}_{2}\right|}\right] \frac{d \mathbf{r}_{1} d \mathbf{r}_{2} d \omega}{2 \pi}
$$

The variational derivative $\delta \chi_{p}^{0} / \delta n_{p}^{l}$ has the simple functional form

$$
\frac{\delta \chi_{p}^{0}\left(\mathbf{r}_{1}, \mathbf{r}_{2}, \omega\right)}{\delta n_{p}^{l}}=\left[G^{p}\left(\mathbf{r}_{1}, \mathbf{r}_{2}, \omega+\varepsilon_{p}^{l}\right)+G^{p}\left(\mathbf{r}_{1}, \mathbf{r}_{2},-\omega+\varepsilon_{p}^{l}\right)\right] \phi_{p}^{l *}\left(\mathbf{r}_{1}\right) \phi_{p}^{l}\left(\mathbf{r}_{2}\right),
$$

where $G^{p}\left(\mathbf{r}_{1}, \mathbf{r}_{2}, \omega\right)$ is the single-particle Green's function for the system of $Z$ noninteracting protons moving in a self-consistent single-particle nuclear potential. The functional derivative $\delta \chi_{p p} / \delta n_{p}^{l}$ is determined by the matrix equation

$$
\frac{\delta \chi_{l m}}{\delta n_{p}^{l}}=\frac{\delta \chi_{l}^{0}}{\delta n_{p}^{l}} \delta_{l m}+\sum_{k}\left[\frac{\delta \chi_{l}^{0}}{\delta n_{p}^{l}} R_{l k} \chi_{k m}+\chi_{l}^{0} \frac{\delta R_{l k}}{\delta n_{p}^{l}} \chi_{k m}+\chi_{l}^{0} R_{l k} \frac{\delta \chi_{k m}}{\delta n_{p}^{l}}\right]
$$

which can be derived by directly varying Eq. (6). Integration with respect to spatial coordinates is implied in Eq. (21) in just the same way as in Eq. (6). As can be seen from Eq. (9), the effective interaction $R_{\alpha \beta}$ is determined by the form of the functional $F_{0}$. In order to simplify the calculations, we took, however, the interaction $R_{\alpha \beta}$ in the separable representation [14],

$$
R_{l k}\left(\mathbf{r}_{1}, \mathbf{r}_{2}\right)=\lambda \frac{d V_{l}\left(r_{1}\right)}{d r} \frac{d V_{k}\left(r_{2}\right)}{d r} \delta\left(\Omega_{1}-\Omega_{2}\right)
$$


where $V_{l}(r)$ is the self-consistent single-particle proton (neutron) potential. The value of the parameter $\lambda$ is chosen in such a way that the dipole response has a pole at $\omega=0$. The above separable form of the effective (residual) interaction is extensively used and, as was shown in [7, 15], provides a good description of collective nuclear excitations. The value calculated here for the shift $\Delta \varepsilon_{p}^{l}$ of single-particle proton levels located near the Fermi level is $(0.2-0.4) \mathrm{MeV}$ both for medium-mass and heavy nuclei, in overall agreement with the value of the Nolen-Schiffer anomaly. It is worthwhile to verify these results by using the simple LDA expressions (14) and (15) for $F^{\text {corr }}\left[\rho_{p}\right]$. The shift $\Delta \varepsilon_{p}^{l}$ then assumes the form

$$
\Delta \varepsilon_{p}^{l}=\int \frac{\delta F_{c}^{c o r r}\left[\rho_{p}\right]}{\delta \rho_{p}}\left|\phi_{p}^{l}(\mathbf{r})\right|^{2} d \mathbf{r}
$$

It is convenient to approximate the single-particle density $\rho_{p}(r)$ by the Fermi distribution

$$
\rho_{p}(r, R)=\frac{\rho_{0}}{1+\exp ((r-R) / a)}
$$

where $R$ is the nuclear radius and the diffuseness parameter $a$ is taken to be $0.6 \mathrm{fm}$. In this case, the functional $F_{c}^{c o r r}\left[\rho_{p}\right]$ given by Eq. (14) reduces to a function $F_{c}^{c o r r}(R)$ of the nuclear radius. An addition of a proton increases the radius $R$ by the quantity $\Delta R$ that is determined from the normalization condition

$$
\int\left[\rho_{p}(r, R+\Delta R)-\rho_{p}(r, R)\right] d \mathbf{r}=1
$$

The CCE-induced shift of a proton level occurring near the Fermi surface is given by

$$
\Delta \varepsilon_{p}=F_{c}^{c o r r}(R+\Delta R)-F_{c}^{c o r r}(R) .
$$

After simple transformations of the relevant integrals of the Fermi functions, we find that, for mediummass and heavy nuclei, the sought shift of a proton level near the Fermi level is

$$
\Delta \varepsilon_{p} \simeq \frac{D a}{2 R} \simeq(0.3-0.4) \mathrm{MeV}
$$

Since corrections of order $D(a / R)^{3}$ were discarded, the result in $(25)$ slightly overestimates the shift.

The calculated mass differences between mirror nuclei are quoted in the table. In this calculation, the functional $F_{0}$ was constructed on the basis of the SIII interaction [8], while the functionals $F_{c}$ and $F_{c}^{c o r r}$ were, respectively, taken in the form (10) and specified by Eqs. (14) and (15).

Mass differences between mirror nuclei (in $\mathrm{MeV}$ )

\begin{tabular}{||l|r|r|r|r|r||}
\hline Nuclei & ${ }^{15} \mathrm{O}-{ }^{15} \mathrm{~N}$ & ${ }^{17} \mathrm{~F}-{ }^{17} \mathrm{O}$ & ${ }^{39} \mathrm{Ca}-{ }^{39} \mathrm{~K}$ & ${ }^{41} \mathrm{Sc}-{ }^{41} \mathrm{Ca}$ & ${ }^{48} \mathrm{Ni}-{ }^{48} \mathrm{Ca}$ \\
\hline Theory & 3.48 & 3.56 & 7.23 & 7.24 & 66.70 \\
\hline Experiment [2,16] & 3.54 & 3.54 & 7.30 & 7.28 & 67.06 \\
\hline
\end{tabular}

The table demonstrates that the mass differences between mirror nuclei are closely reproduced. The remaining disagreement can be used to determine the coupling constants for forces violating the charge symmetry of nuclear interaction. Thus, traditional nuclear physics can be advantageous 
in calculating the constants concerning elementary-particle physics. Equation (25) shows that the shift $\Delta \varepsilon_{p}$ of a single-particle level approximately compensates for the level shift caused by exchange Coulomb interaction. It should be recalled that the exchange Coulomb interaction reduces the energy of a single-particle level, as can be seen from Eq. (4). Therefore, the total shift of a single-particle level due to Coulomb interaction can be derived by taking into account solely the direct Coulomb interaction - that is, by retaining only the term $F_{c}^{H}$ in Eq. (10). A similar procedure was postulated in [9,16]. It should be noted that the recipe assuming mutual cancellation of the exchange Coulomb interaction and the CCE does not lead to a noticeable value of the coefficient $a_{2}^{\prime}$ in Eq. (17) (recall this coefficient determines the isovector component of the surface tension) because the contribution from $F_{c}^{H}$ and $F_{c}^{F}$ [see Eq. (4)] to the surface tension is small. On the contrary, our calculations indicate that the coefficient $a_{2}^{\prime}$ is not small. It also follows from Eq. (25) that, upon taking the CCE into account, the last filled single-particle proton level is shifted upward by 0.3 to $0.4 \mathrm{MeV}$. As a result, the maximal charge $Z$ of a nucleus occurring near the proton drip line decreases by 2 or 3 units, as follows from [17]. Therefore, we conclude that, in calculating the proton drip line, it is important to take the CCE into account.

\section{EFFECTIVE MASS}

Let us consider the change $\Delta M$ that Coulomb interaction induces in the effective proton mass $M^{*}$. In the case of uniform nuclear matter, the single-particle spectrum depends on the momentum $p$, while the effective mass, as follows from Eq. (18), is determined by the expression

$$
\frac{1}{M^{*}}=\left.\frac{1}{p_{F}} \frac{d \varepsilon_{p}(p)}{d p}\right|_{p=p_{F}}
$$

where $p_{F}$ is the Fermi momentum. In order to calculate $\Delta M$, we use Eq. (26), replacing $\varepsilon_{p}(p)$ by the shift of the single-particle spectrum due to Coulomb interaction. We have

$$
\frac{\Delta M}{M^{*}\left(M^{*}+\Delta M\right)}=\frac{e^{2}}{p_{F}} \frac{d}{d p} \frac{\delta}{\delta n_{p}} \int\left[\frac{\chi_{p p}(q, i \omega)+2 \pi \rho_{p} \delta(\omega)}{q^{2}}\right] \frac{d q d \omega}{(2 \pi)^{3}} .
$$

Evaluating variations in Eq. (27) and considering that, in the case of uniform matter, Eqs. (6) and (21) reduce to algebraic equations, we obtain [14,18],

$$
\frac{\Delta M}{M^{*}\left(M^{*}+\Delta M\right)}=\frac{e^{2} d}{p_{F} d p} \int \frac{\delta \chi_{0}(q, i \omega)}{\delta n_{p}} \frac{1}{\left(1-R_{+}(q, i \omega) \chi_{0}(q, i \omega)\right)^{2}} \frac{d \mathbf{q} d \omega}{q^{2}(2 \pi)^{3}}
$$

where $M^{*}$ is the effective proton mass in the absence of Coulomb interaction and $R_{+}=\left(R_{p p}+R_{p n}\right) / 2$ is the effective interaction. The derivative $d / d p$ was taken at the point $p=p_{F}$. Let us consider the effective-mass variation $\Delta M$ for the case where the system under consideration is characterized by the compressibility parameter $\kappa, 1 / \kappa=\rho^{2} d^{2} E / d \rho^{2}$, tending to infinity. This state is similar to states existing in the surface region of nuclei. This consideration will enable us to draw qualitative conclusions on the behavior of the effective mass in a nucleus. It can be shown that [18]

$$
\left.\frac{d}{d p} \frac{\delta}{\delta n_{p}} \chi_{0}(q, \omega)\right|_{p \rightarrow p_{F}}=-\frac{4 \pi}{p_{F}^{2}} \delta\left(p_{F}-|\mathbf{p}+\mathbf{q}|\right) \delta(\omega) \mathbf{p}(\mathbf{p}+\mathbf{q}) .
$$


Substituting (29) into (28) and retaining only the leading term, we obtain

$$
\frac{1}{M^{*}+\Delta M}=\frac{1}{M^{*}}+\frac{e^{2}}{2 \pi p_{F}} \int_{-1}^{1} \frac{x d x}{(1-x)\left[1-R(q(x), 0) \chi_{0}(q(x), 0)\right]^{2}} .
$$

where $q(x)=p_{F} \sqrt{2(1-x)}$. At the point where the compressibility diverges, the denominator $(1-$ $\left.R_{+} \chi_{0}\right)^{2}$ of the integrand in Eq. (30) vanishes at $x=1(q=0)$. The relevant integral, which is positive, diverges, which results in the vanishing of the effective mass - that is, $M^{*}+\Delta M \rightarrow 0$. This result demonstrates that, in equilibrium nuclear systems, the CCE noticeably affects the effective mass, the decrease $\Delta M$ in the effective proton mass being dependent on the degree to which the density of the system, $\rho$, deviates from its equilibrium value $\rho_{0}$. In a nucleus, the divergence of the integral in Eq. (30) - it could be due to the decrease in the density near the surface of the nucleus - gives way to a finite value. As a result, the effective proton mass decreases in relation to $M^{*}$ and becomes smaller than the effective neutron mass. It can be said that the effective nucleon mass acquires an isovector component [14]. This possibility was discussed in [16]. A theoretical validation of this effect has been given here.

\section{CONCLUSION}

The basic results of the present study can be summarized as follows. A consistent scheme for constructing the density functional determining the Coulomb energy of a nucleus has been developed to the first order in $e^{2}$. Using this functional, we have calculated the single-particle spectra of nuclei and the systematic shift of these spectra that is induced by the CCE. It has been shown that the Nolen-Schiffer anomaly is removed to a considerable extent by this systematic shift of the singleparticle spectrum. Owing to the same mechanism, the proton drip line undergoes a shift of 2 or 3 units toward smaller values of the charge $Z$ of a nucleus occurring near this line. It has been shown that the CCE must be taken into account in calculating the effective nucleon mass. This opens the possibility of estimating the coupling constants for forces violating charge symmetry. The contribution of these forces must be treated in the same manner as this has been done for the Coulomb interaction. It can be expected that the proposed procedure will make it possible to construct a density functional applicable to describing various properties of nuclei occurring both in the valley of stability and beyond it.

\section{ACKOWLEDGMENTS}

I am grateful to A. Bulgac for stimulating discussions on the problems analyzed in this study. This work was supported in part by INTAS-OPEN-97-603. 


\section{REFERENCES}

[1] J.A. Nolen, and J.P. Schiffer, Annu. Rev. Nucl. Sci. 19, 4711964.

[2] S. Shlomo, Rep. Prog. Phys. 41, 957 (1978); S. Shlomo, and M.G. Love, Phys. Scr. 26, 280 (1982).

[3] N. Auerbach, Phys. Rep. 98, 273 (1983).

[4] G.A. Miller, B.M.K. Nefkens, and I. Slaus, Phys. Rep. 194, 1 (1990).

[5] V. R. Shaginyan, Yad. Fiz. 39, 346 (1984) [Sov. J. Nucl. Phys. 39, 218 (1984)]; 40, 1144 (1984) [40, $728(1984)]$.

[6] A. Bulgac, and V.R. Shaginyan, JETP Lett. 62, 843 (1995); A. Bulgac, and V.R. Shaginyan, Nucl. Phys. A 601, 103 (1996).

[7] P. Ring, and P. Schuck, Nuclear Many Body Problem (New York, Springer-Verlag, 1980).

[8] D. Vautherin, and D.M. Brink, Phys. Rev. C 5, 626 (1972); M. Beiner, H. Flocard, Ngueyn van Giai, and P. Quentin, Nucl. Phys. A 238, 29 (1975).

[9] S.A. Fayans, JETP Lett. 68, 169 (1998).

[10] P. Hohenberg, and W. Kohn, Phys. Rev. B 136, 864 (1965); W. Kohn, and L.J. Sham, Rev. A 140, 1133 (1965).

[11] A. B. Migdal, Theory of Finite Fermi Systems and Applications to Atomic Nuclei (Nauka, Moscow, 1965; Interscience, New York, 1967).

[12] H.A. Bethe, Annu. Rev. Nucl. Sci. 93, 93 (1971).

[13] L. D. Landau, Zh. Eksp. Teor. Fiz. 30, 1058 (1956) [Sov. Phys. JETP 3, 920 (1956)].

[14] A. Bulgac, and V.R. Shaginyan, Eur. Phys. J. A 5, 247 (1999); A. Bulgac, and V.R. Shaginyan, Phys. Lett. B 469, 1 (1999).

[15] A. Bohr and B. R. Mottelson, Nuclear Structure Vol. 2: Nuclear Deformations (Benjamin, New York, 1975).

[16] B.A. Brown, Phys. Rev. C 58, 220 (1998).

[17] A. Bohr and B. R. Mottelson, Nuclear Structure, Vol. 1: Single-Particle Motion (Benjamin, New York, 1969), Fig. 2.30.

[18] V.A. Khodel, V.R. Shaginyan, and M.V. Zverev, JETP Lett. 65, 253 (1997). 